This item was submitted to Loughborough's Research Repository by the author.

Items in Figshare are protected by copyright, with all rights reserved, unless otherwise indicated.

\title{
Full vehicle and tyre identification using unscented and extended identifying Kalman Filters
}

PLEASE CITE THE PUBLISHED VERSION

http://dx.doi.org/10.1201/9781315265285-79

PUBLISHER

(C) Taylor and Francis

\section{VERSION}

AM (Accepted Manuscript)

\section{PUBLISHER STATEMENT}

This work is made available according to the conditions of the Creative Commons Attribution-NonCommercialNoDerivatives 4.0 International (CC BY-NC-ND 4.0) licence. Full details of this licence are available at: https://creativecommons.org/licenses/by-nc-nd/4.0/

\section{LICENCE}

CC BY-NC-ND 4.0

\section{REPOSITORY RECORD}

Best, Matt C., and Karol Bogdanski. 2016. "Full Vehicle and Tyre Identification Using Unscented and Extended Identifying Kalman Filters". figshare. https://hdl.handle.net/2134/22450. 


\title{
Full Vehicle and Tyre Identification using Unscented and Extended Identifying Kalman Filters
}

\author{
Matthew C Best and Karol Bogdanski \\ Loughborough University \\ E-mail: M.C.Best@lboro.ac.uk
}

\begin{abstract}
This paper considers identification of all significant vehicle handling and driveline dynamics of a test vehicle, including identification of a combined-slip tyre model, using only those sensors currently available on most vehicle CAN buses. The method extends previous work using augmented Kalman Filter state estimators to concentrate wholly on parameter identification, and it compares Extended and Unscented Kalman filter algorithms. Using an appropriately simple but efficient model structure, all of the independent parameters are found from test vehicle data, with the resulting model accuracy demonstrated on independent validation data. The method is suited to applications of system identification, but also in on-line model predictive controllers or estimators. It can also operate in real-time, so the model could be continuously identified to maintain accuracy with each new journey.
\end{abstract}

Topics / Vehicle Dynamics, Tyre Model, Modeling and Simulation, State Estimation, System Identification

\section{INTRODUCTION}

Several previous publications have considered estimation of individual parameters of a vehicle dynamic model, particularly for tyre and friction coefficient estimation [1,2]. The validity of these (necessarily simplified) models also depends on many other fixed, estimated parameters. Usually, even if these other values are physically accurately set, the simplified model can be made to perform better if they are tuned or also identified.

Here we embark on an ambitious attempt to identify all the independent parameters in a simplified whole vehicle handling model, including yaw and roll freedoms, independent combined-slip load dependent tyres and appropriate drivetrain lags. This is achievable, given recent findings that Kalman filter methods can be applied to identify all parameters in any wellconditioned model structure [3].

In the extended abstract we demonstrated the principle by simulated identification of longitudinal tyre dynamics, including wheel-spin and lock, using an Extended Kalman Filter. In this final paper we consider data collected from a test vehicle carrying out medium to high magnitude manoeuvres including wheel-spin and terminal understeer, in order to build a model which is valid over the whole range of the tyres. We also consider the relative advantages over EKF of using the more computationally efficient, Unscented Kalman Filter for the identification process.

\section{IDENTIFYING EXTENDED KALMAN FILTER}

The standard Extended Kalman Filter (EKF) operates on nonlinear system and sensor models $\mathbf{f}$ and $\mathbf{h}$, which relate the state vector $\mathbf{x}$, measured sensor set $\mathbf{y}$, known inputs $\mathbf{u}$ and model parameters $\boldsymbol{\theta}$ through

$$
\begin{array}{r}
\dot{\mathbf{x}}=\mathbf{f}(\mathbf{x}, \mathbf{u}, \boldsymbol{\theta})+\boldsymbol{\omega} \\
\mathbf{y}=\mathbf{h}(\mathbf{x}, \mathbf{u}, \boldsymbol{\theta})+\mathbf{v}
\end{array}
$$

$\boldsymbol{\omega}$ represents state propagation and modelling error, $v$ is the sensor error, and an optimal filter can be derived using estimates or expectations of the error covariance matrices :

$$
\mathbf{Q}=E\left(\boldsymbol{\omega} \boldsymbol{\omega}^{T}\right) \quad \mathbf{R}=E\left(\boldsymbol{v} \boldsymbol{v}^{T}\right)
$$

The EKF also requires model Jacobians to be evaluated at each time step, defined

$$
\mathbf{F}=\frac{\partial \mathbf{f}(\mathbf{x}, \mathbf{u}, \boldsymbol{\theta})}{\partial \mathbf{x}} \quad \mathbf{H}=\frac{\partial \mathbf{h}(\mathbf{x}, \mathbf{u}, \boldsymbol{\theta})}{\partial \mathbf{x}}
$$

For further details of the full EKF see [2].

The Identifying EKF (IEKF) takes advantage of the fact that $\mathbf{f}$ and $\mathbf{h}$ are general nonlinear functions of $\mathbf{x}$ and $\boldsymbol{\theta}$, defining an extended state vector $\mathbf{z}$ with extended state derivatives set zero for the parameter states :

$$
\dot{\mathbf{z}}=\left[\begin{array}{c}
\dot{\mathbf{x}} \\
\dot{\boldsymbol{\theta}}
\end{array}\right]=\left[\begin{array}{c}
\mathbf{f}(\mathbf{z}, \mathbf{u}) \\
\mathbf{0}
\end{array}\right]
$$

It is computed using a sequence of equations which develop a time-varying estimate of state error covariance, $\mathbf{P}_{k}$ and Kalman gain $\mathbf{K}_{k}$; at each time step of the recorded time histories, compute

$$
\begin{aligned}
\mathbf{K}_{k}= & \mathbf{P}_{k} \mathbf{H}_{k}^{\mathbf{T}}\left[\mathbf{H}_{k} \mathbf{P}_{k} \mathbf{H}_{k}^{\mathrm{T}}+\mathbf{R}\right]^{-\mathbf{1}} \\
& \mathbf{P}_{k}^{*}=\left[\mathbf{I}-\mathbf{K}_{k} \mathbf{H}_{k}\right] \mathbf{P}_{k} \\
\mathbf{P}_{k+1}= & \mathbf{P}_{k}^{*}+T\left[\mathbf{F}_{k} \mathbf{P}_{k}^{*}+\mathbf{P}_{k}^{*} \mathbf{F}_{k}^{\mathbf{T}}+\mathbf{Q}\right] \\
\hat{\mathbf{z}}_{k+1}= & \hat{\mathbf{z}}_{k}+T \mathbf{f}_{k}+\mathbf{K}_{k}\left(\mathbf{y}_{k}-\mathbf{h}_{k}\right)
\end{aligned}
$$


The final equation in set (6) combines Euler integration of the system using time step $T$ with state and parameter adaptation driven by the output error, (known as the innovation sequence).

\section{UNSCENTED KALMAN FILTER}

The unscented Kalman filter (UKF) identifies its own error statistics at each iteration, and hence avoids the need to use Jacobians. According to [4],[5], a sample of $(2 n+1)$ so called sigma points are selected around the $n^{\text {th }}$ order state vector, at each instant $k$ in time:

$$
\begin{aligned}
\chi_{0 k} & =\hat{\mathbf{x}}_{k} \\
\chi_{i k} & =\hat{\mathbf{x}}_{k}+\left\{\sqrt{(n+\kappa) \mathbf{P}_{k}}\right\}_{i} \\
\chi_{(i+n) k} & =\hat{\mathbf{x}}_{k}-\left\{\sqrt{(n+\kappa) \mathbf{P}_{k}}\right\}_{i}
\end{aligned}
$$

where $\left\{\sqrt{(n+\kappa) \mathbf{P}_{k}}\right\}_{i}$ is the $\mathrm{i}^{\text {th }}$ column of the matrix square root of $(n+\kappa) \mathbf{P}_{k}$ (obtained here using Cholesky decomposition) and $\mathbf{P}_{\mathbf{k}}$ is the current estimate of state error covariance. These sigma points are propagated by the model, here as for IEKF, using Euler approximation:

$$
\chi_{i(k+1)}=T \mathbf{f}\left(\chi_{i k}, \mathbf{u}_{k}, \boldsymbol{\theta}\right)
$$

and intermediate estimates for the propagated state and covariance matrix are computed by weighted averages:

$$
\begin{gathered}
\hat{\mathbf{x}}_{k+1}^{*}=\sum_{i=0-2 n} W_{i} \boldsymbol{\chi}_{i(k+1)} \\
\mathbf{P}_{k+1}^{*}=\sum_{i=0-2 n} W_{i}\left\{\boldsymbol{\chi}_{i(k+1)}-\hat{\mathbf{x}}_{(k+1)}\right\}\left\{\boldsymbol{\chi}_{i(k+1)}-\hat{\mathbf{x}}_{(k+1)}\right\}^{T}+T \mathbf{Q}
\end{gathered}
$$

With $W_{0}=\frac{\kappa}{n+\kappa}$ and for all other $i, W_{i}=\frac{1}{2(n+\kappa)}$

Similarly, average outputs are obtained according to the output model :

$$
\begin{aligned}
& \Upsilon_{i(k+1)}=\mathbf{h}\left(\boldsymbol{\chi}_{i k}, \mathbf{u}_{k}, \boldsymbol{\theta}\right) \\
& \hat{\mathbf{y}}_{k+1}=\sum_{i=0-2 n} W_{i} \Upsilon_{i(k+1)}
\end{aligned}
$$

The UKF then propagates output error covariance according to the transformed sigma points:

$$
\mathbf{P}_{y y}=\sum_{i=0-2 n} W_{i}\left\{\Upsilon_{i(k+1)}-\hat{\mathbf{y}}_{(k+1)}\right\}\left\{\Upsilon_{i(k+1)}-\hat{\mathbf{y}}_{(k+1)}\right\}^{T}+\mathbf{R}
$$

and uses this together with a cross correlation estimate

$$
\mathbf{P}_{x y}=\sum_{i=0-2 n} W_{i}\left\{\boldsymbol{\chi}_{i(k+1)}-\hat{\mathbf{x}}_{(k+1)}\right\}\left\{\Upsilon_{i(k+1)}-\hat{\mathbf{y}}_{(k+1)}\right\}^{T}
$$

to find the Kalman gain by

$$
\mathbf{K}_{k+1}=\mathbf{P}_{x y} \mathbf{P}_{y y}^{-1}
$$

State and covariance estimates are then updated using the innovation sequence, in a similar way to IEKF:

$$
\begin{array}{r}
\mathbf{P}_{k+1}=\mathbf{P}_{k+1}^{*}-\mathbf{K}_{k+1} \mathbf{P}_{y y} \mathbf{K}_{k+1}^{T} \\
\hat{\mathbf{x}}_{k+1}=\hat{\mathbf{x}}_{k+1}^{*}+\mathbf{K}_{k+1}\left(\mathbf{y}_{k+1}-\hat{\mathbf{y}}_{k+1}\right)
\end{array}
$$

The above UKF derivation is common to all implementations of state and parameter estimation, so the simple substitution of $\mathbf{x}$ in the above, with $\mathbf{z}$ from eqn (5) provides the identifying filter.

\section{VEHICLE MODEL}

The vehicle model is based on the well-known three degree of freedom model, simulating yaw, roll, and sideslip using a load dependent, combined-slip Pacejka tyre model. A fourth, longitudinal degree of freedom is included, so the principal equations of motion are

longitudinal: $M \dot{U}=\sum_{i=1,4} F_{x i}+M r V+M h r p$

lateral:

$$
M \dot{V}+M h \dot{p}=\sum_{i=1,4} F_{y i}-M U r
$$

yaw:

$$
I_{z z} \dot{r}=a \sum_{i=1,2} F_{y i}-b \sum_{i=3,4} F_{y i}+c \sum_{i=1,3} F_{x i}-c \sum_{i=2,4} F_{x i}
$$

roll:

$$
\begin{aligned}
& M h \dot{V}+I_{x x} \dot{p}=\cdots \\
& -M h U r+M g h \phi-h_{0} \sum_{i=1-4} F_{y i}+c \sum_{i=1,3} F_{z}-c \sum_{i=2,4} F_{z}
\end{aligned}
$$

roll kinematics:

$$
\dot{\varphi}=p
$$

Standard SAE axes are used, fixed relative to the vehicle wheelbase, and the wheels are labelled (1-4) in ascending order as (front-left, front-right, rear-left, rearright). Equal half-track $c$ is assumed, with axles separated distances $a$ and $b$ from the front and rear axles respectively, and vertical suspension geometry is based on fixed roll centres $h_{R(f, r)}$ and CG height $h_{G}$ such that

$$
h_{0}=h_{R f}+\frac{W_{r}}{M g}\left(h_{R r}-h_{R f}\right) \quad h=h_{G}-h_{0}
$$

The forces controlling the vehicle body motion $\left(F_{x i}, F_{y i}\right)$ allow for large steer angles

$$
\begin{array}{ll}
F_{x 1,2}=F_{t x 1,2}^{*} \cos \delta-F_{t y 1,2}^{*} \sin \delta & F_{x 3,4}=F_{t x 3,4}^{*} \\
F_{y 1,2}=F_{t y 1,2}^{*} \cos \delta-F_{t x 1,2}^{*} \sin \delta & F_{y 3,4}=F_{t y 3,4}^{*}
\end{array}
$$

based on lagged tyre forces, where each of the 8 elements are lagged to simulate relaxation within the tyre

$$
\dot{F}_{t x / y, i}^{*}=\tau^{-1}\left(F_{t x / y, i}-F_{t x / y, i}^{*}\right)
$$

The tyre forces $\left(F_{t x i}, F_{t y i}\right)$ are modelled according to a slightly simplified Pacejka magic formula

$$
P(|\mathbf{k}|)=D \sin \left(C \tan ^{-1}\left(\frac{|\mathbf{k}|}{C}-E\left(\frac{|\mathbf{k}|}{C}-\tan ^{-1} \frac{|\mathbf{k}|}{C}\right)\right)\right)(22
$$

using normalized slip and isotropic similarity scaling [6],[7]. The normalized slip vector is 


$$
\mathbf{k}=\left(\begin{array}{l}
k_{x} \\
k_{y}
\end{array}\right)=\frac{C_{\alpha}}{\mu F_{z}}\left(\begin{array}{c}
K_{x} S_{i} \\
\tan \alpha_{i}
\end{array}\right)
$$

where $S$ is the longitudinal slip ratio, and $\alpha$ is the slip angle at each tyre contact patch,

$$
S_{i}=\frac{r_{r} \omega_{i}-U_{x i}}{U_{x i}} \quad \tan \alpha_{i}=\frac{-U_{y i}}{U_{x i}}
$$

based on wheel oriented velocities

$$
\begin{aligned}
& U_{x i}=U_{x i}^{*} \cos \delta_{i}+U_{y i}^{*} \sin \delta_{i} \\
& U_{y i}=U_{y i}^{*} \cos \delta_{i}-U_{x i}^{*} \sin \delta_{i}
\end{aligned}
$$

and

$$
\begin{array}{cc}
U_{x 1,3}^{*}=U+c r & U_{x 2,4}^{*}=U-c r \\
U_{y 1,2}^{*}=V+a r & U_{y 3,4}^{*}=V-b r
\end{array}
$$

The resulting tyre force vector is then

$$
\left(\begin{array}{c}
F_{t x} \\
F_{t y}
\end{array}\right)=P(|\mathbf{k}|) \frac{\mu F_{z}}{|\mathbf{k}|}\left(\begin{array}{l}
k_{x} \\
k_{y}
\end{array}\right)
$$

Vertical tyre loads $F_{z}$ are calculated from static weight distribution, modified to accommodate lateral load transfer using separate front/rear distributions according to :

$$
\Delta_{\text {lat }} F_{z(f / r)}=\frac{\sum F_{y(f / r)} h_{R(f / r)}+K_{\phi(f / r)} \phi+B_{\phi(f / r)} p}{2 c}
$$

and longitudinal load transfer, according to a rigid, zero pitch approximation

$$
\Delta_{\text {long }} F_{z}=\frac{\sum_{i=1-4} F_{x i} h_{G}}{(a+b)}
$$

\section{IMPLEMENTATION}

Test data were obtained from a '08 MY Jaguar XF equipped with an OXTS 3200 inertial navigation (IN) device, driven on a dry proving ground. For the identification, input data is comprised of steer angle and the four wheel speeds, and the outputs are CG longitudinal and lateral accelerations and body roll rate, modeled as

$$
\begin{aligned}
& \mathbf{u}=\left[\delta, \omega_{1}, \omega_{2}, \omega_{3}, \omega_{4}\right]^{T} \\
& \mathbf{y}=\left[\frac{1}{M} \sum_{i=1-4} F_{x i}, \frac{1}{M} \sum_{i=1-4} F_{x i}, p\right]^{T}
\end{aligned}
$$

All the above measurements with the exception of body roll rate were taken from the vehicle CAN; although roll was measured using the IN, this sensor may already be available in other CAN sets, or would be cheap to add. IN measured vehicle speed, roll angle, yaw rate and lateral velocity are also used for validation of the identified model.

A range of high and low magnitude, separated and combined slip manoeuvres were conducted, exciting both dynamic and steady-state vehicle responses. One 100 second test covering dynamic, combined excitation of steering, brake and acceleration inputs was used as the identification set, with a variety of other tests used for validation. The CAN data was inter-sampled at a constant $100 \mathrm{~Hz}$ to match the IN (setting $T=0.01$ ) and all data was then digitally filtered at $10 \mathrm{~Hz}$ to remove higher frequency noise; this is necessary to prevent instability in both Kalman filters, as they are propagated by Euler integration. Although here we perform a batch data process, the identifying KFs are designed to work equally well in real-time, and real-time parameter tuning could easily be achieved using a combination of dynamic low pass filters and/or higher sampling rate $T$.

Identification is achieved iteratively by 'rinsing' the data repeatedly through either Kalman filter, starting with an initial, nominal parameter set. Both filters require covariance estimates and here $\mathbf{Q}$ is fixed throughout as $\mathbf{Q}=\rho \mathbf{I}$, with $\rho=1 \mathrm{e}-7$ (see [3] for more detail). For any given estimate $\hat{\boldsymbol{\theta}}$ of the identified parameters, $\mathbf{R}$ can be obtained numerically from the covariance of $v$ using eqn (2). Here, $\mathbf{R}$ is recomputed after each iteration. Finally, $\mathbf{P}_{\mathbf{0}}=\mathbf{Q}$, and for the UKF $\kappa=1$.

The only difficult task is the decision of which parameters to adapt in the identification, and which to fix. The KFs can identify any number of parameters, but if they are not independent in their influence on the recorded outputs, the parameters will diverge. Following various trials the following identification set was established as the minimum non-divergent set:

$$
\begin{aligned}
& \boldsymbol{\theta}_{\text {id }}=\left[h_{G}, I_{z z}, K_{\phi}, B_{\phi}, K_{x f}, K_{x r}, C_{\alpha f}, C_{\alpha r}, \cdots\right. \\
& \left.C_{f}, D_{f}, E_{f}, C_{r}, D_{r}, E_{r}\right]
\end{aligned}
$$

This allows full identification of separate front and rear individual tyre-suspension models but with roll stiffness and damping applied at a constant ratio, known from manufacturer supplied data. The remaining fixed parameters constrain the weight balance, roll inertia and essential geometry ( $L=$ wheelbase), and are set

$$
\begin{aligned}
& \boldsymbol{\theta}_{\text {fix }}=\left[W_{f}=9567, W_{r}=8635, I_{x x}=696, \cdots\right. \\
& \left.h_{R f}=0.08, h_{R r}=0.125, c=0.79, L=2.91\right]
\end{aligned}
$$

Friction is identified via the tyre $D$ parameters, so we set $\mu=1$. To illustrate the effect of identifying too many parameters, we will also consider the case of adding roll centre heights $h_{R f}$ and $h_{R r}$ to the identified set; these are referred to below as the $h+$ case.

\section{RESULTS}

Fig. 1 and Table 1 illustrate convergence results over 200 passes (iterations) through the 100 seconds of identification data for IEKF, UKF and h+ (IEKF) cases. The resulting parameters are generally in the range expected, with the front tyre having lower stiffness and quicker saturation than the rear; this is consistent with expected front steer compliance seen in an earlier study [8].

Most striking is that although the methods are very different, the IEKF and UKF results are almost identical, not just in the final model, but throughout the 
convergence process; only the magnitude of trace $\left(\mathbf{P}_{\mathrm{k}}\right)$ varies between them.

The decision over which of IEKF and UKF is 'better' therefore depends only on computational efficiency and complexity of code. UKF has the advantage that Jacobians are not required, so it is simpler to code, though Matlab's Symbolic toolbox means this overhead in complexity is slight. The CPU time needed to evaluate $\mathbf{F}$ and $\mathbf{H}$ might make the UKF preferable, and this is likely to be significant for state estimation applications where the state vector is smaller. Here the long state vector $\mathbf{z}$ means the UKF computes 55 sigma points at each time step, so it does not operate much quicker than IEKF; for each iteration UKF took 212 seconds, where IEKF took 266. We can therefore conclude UKF is the better method, being simpler and faster, though this may not be the case if the parameter count rose significantly.

The $\mathrm{h}+$ case sees a slight performance advantage in roll rate, but this is at the expense of a converged, physically interpretable model. Note that the result in Table 1 is a snapshot at the $200^{\text {th }}$ iteration, showing unrealistic negative $h_{R f}$ and high $K_{\phi}$ and $h_{G}$; the parameters are also divergent, with $K_{\phi}$ and $h_{G}$ both rising. Interestingly, the 'snapshot' $h+$ model still delivers excellent performance across all outputs (see below).
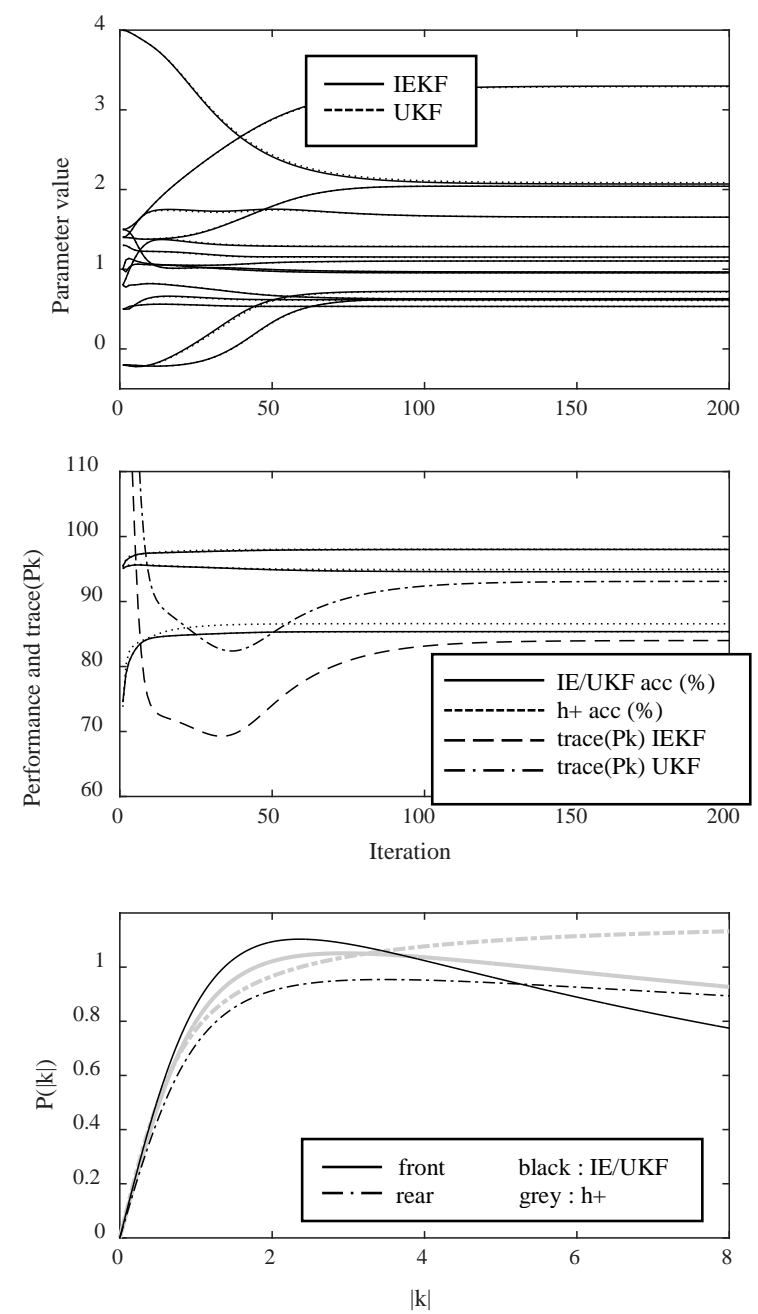

Fig. 1: Identification convergence and resulting tyre
Table 1 : Parameters identified

\begin{tabular}{|l|c|c|c|}
\hline Parameter & IEKF & UKF & h+ \\
\hline$h_{G}(\mathrm{~m})$ & 0.533 & 0.532 & 0.633 \\
\hline$h_{R f}(\mathrm{~m})$ & $(0.08)$ & $(0.08)$ & -0.344 \\
\hline$h_{R r}(\mathrm{~m})$ & $(0.125)$ & $(0.125)$ & 0.223 \\
\hline$I_{z z}\left(\mathrm{x} 1000 \mathrm{kgm}^{2}\right)$ & 2.069 & 2.086 & 2.214 \\
\hline$K_{\phi}(\mathrm{x} 100 \mathrm{kNm} / \mathrm{rad})$ & 1.152 & 1.147 & 1.692 \\
\hline$B_{\phi}(\mathrm{x} 10 \mathrm{kNms} / \mathrm{rad})$ & 0.615 & 0.608 & 1.010 \\
\hline$K_{x f}$ & 3.299 & 3.291 & 3.116 \\
\hline$K_{x r}$ & 0.966 & 0.966 & 1.036 \\
\hline$C_{\alpha f}(\mathrm{x} 100 \mathrm{kN} / \mathrm{rad})$ & 0.625 & 0.627 & 0.665 \\
\hline$C_{\alpha r}(\mathrm{x} 100 \mathrm{kN} / \mathrm{rad})$ & 1.283 & 1.286 & 1.311 \\
\hline$C_{f}$ & 2.042 & 2.043 & 1.801 \\
\hline$D_{f}$ & 1.103 & 1.102 & 1.051 \\
\hline$E_{f}$ & 0.630 & 0.632 & 0.724 \\
\hline$C_{r}$ & 1.653 & 1.652 & 1.085 \\
\hline$D_{r}$ & 0.954 & 0.955 & 1.155 \\
\hline$E_{r}$ & 0.720 & 0.714 & 0.711 \\
\hline
\end{tabular}
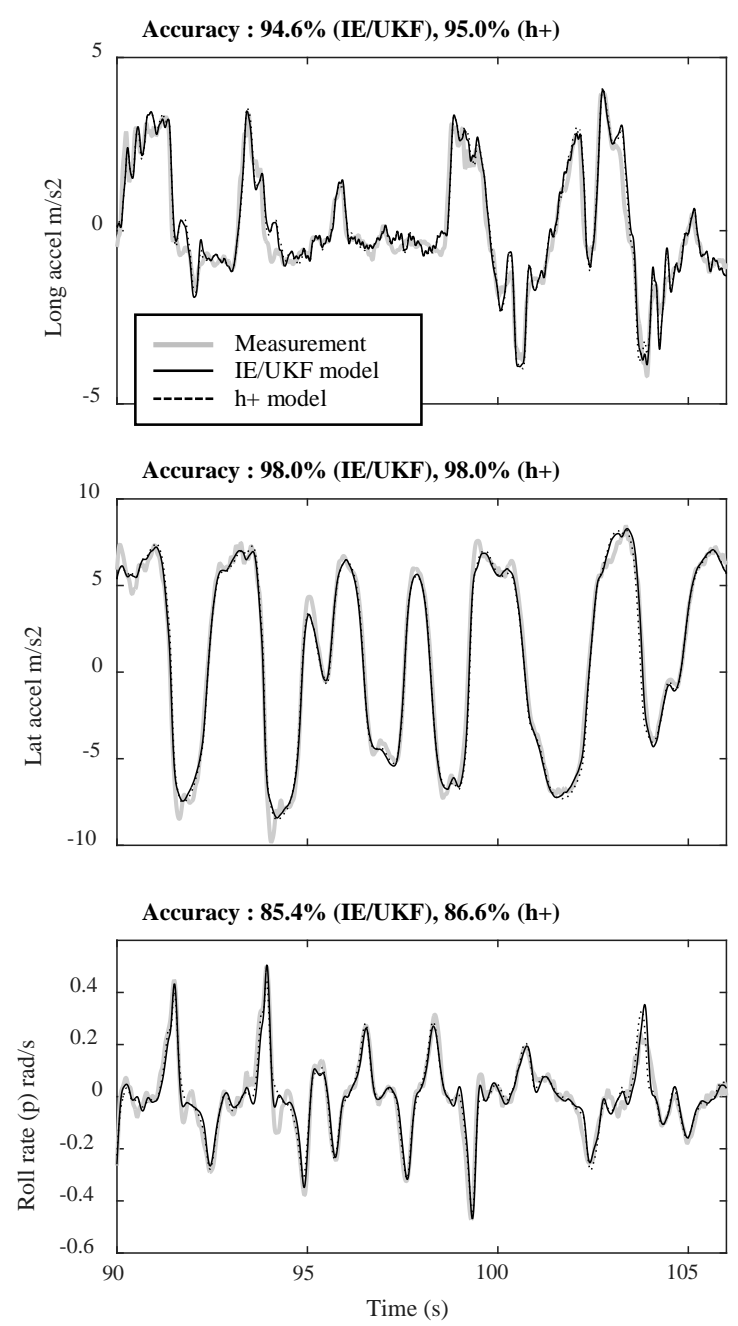

Fig. 2: Model fit to measured, fitted output data 

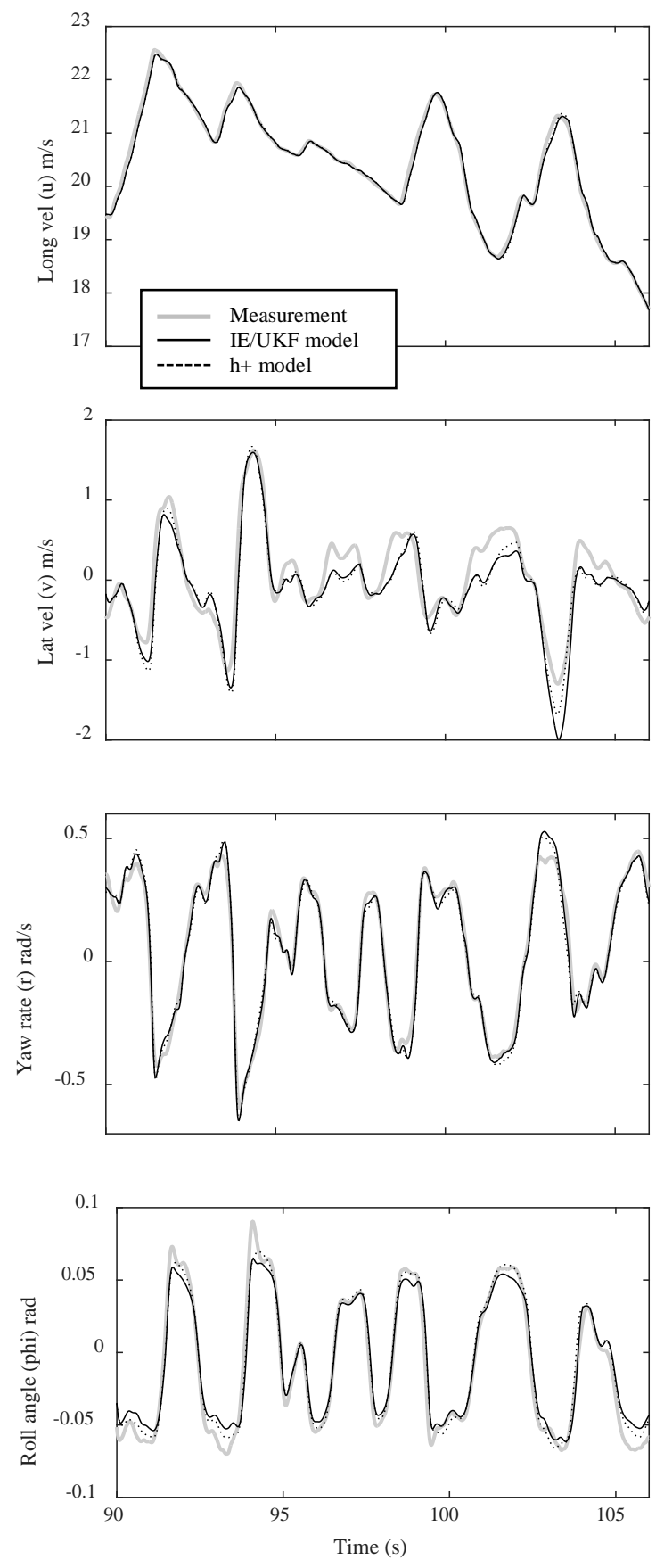

Fig. 3: Model fit to unfitted measurements in the identification data

Fig. 2 shows performance of the identified models on the fitted outputs and Fig. 3 shows good fits to additional unfitted outputs that were also measured.

Validation of the models on an independent test, a step steer followed by combined braking, is presented in fig. 4. Some error is introduced to lateral acceleration when the brake is applied, at 17-18 seconds, but the model is still acceptable. Sadly there was a problem with calibration of the lateral velocity sensor - fig. 4 shows that the model matches the essential dynamic behavior, but the measurement shows drift error over the first 10 seconds of this test, where the vehicle actually followed a straight path.
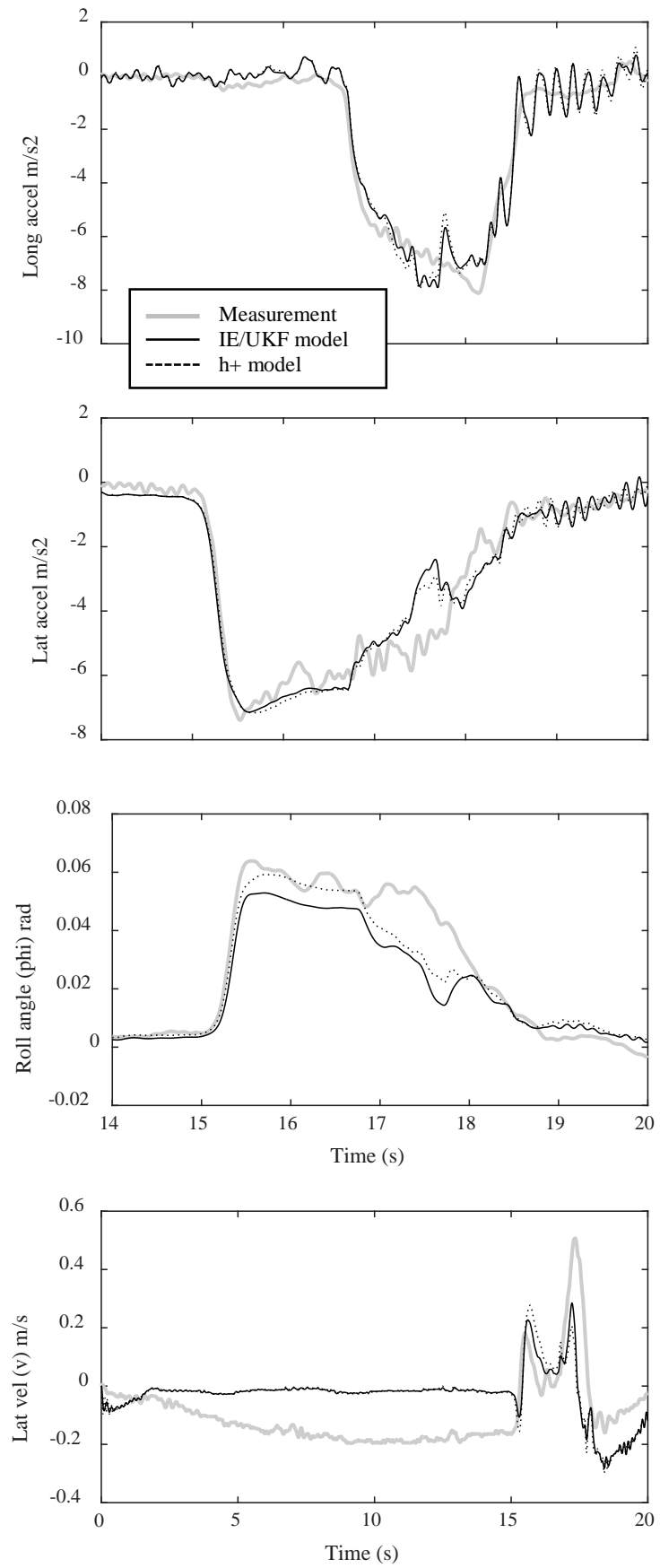

Fig. 4: Identified model fit to validation test

Finally, figs 5 and 6 show a very positive result, validating the combined-slip tyre/suspension model in single-slip tests. Here we see very accurate results for a straight line braking and acceleration test under zero steer; the modeled longitudinal acceleration is indistinguishable from the measured data despite the high $k_{x}$ excursions caused by wheel-spin. And fig. 6 sees high accuracy for a constant speed, random steer manoeuvre. Both of these tests explore the tyre in low to medium magnitude and also in over-slip saturation conditions. 

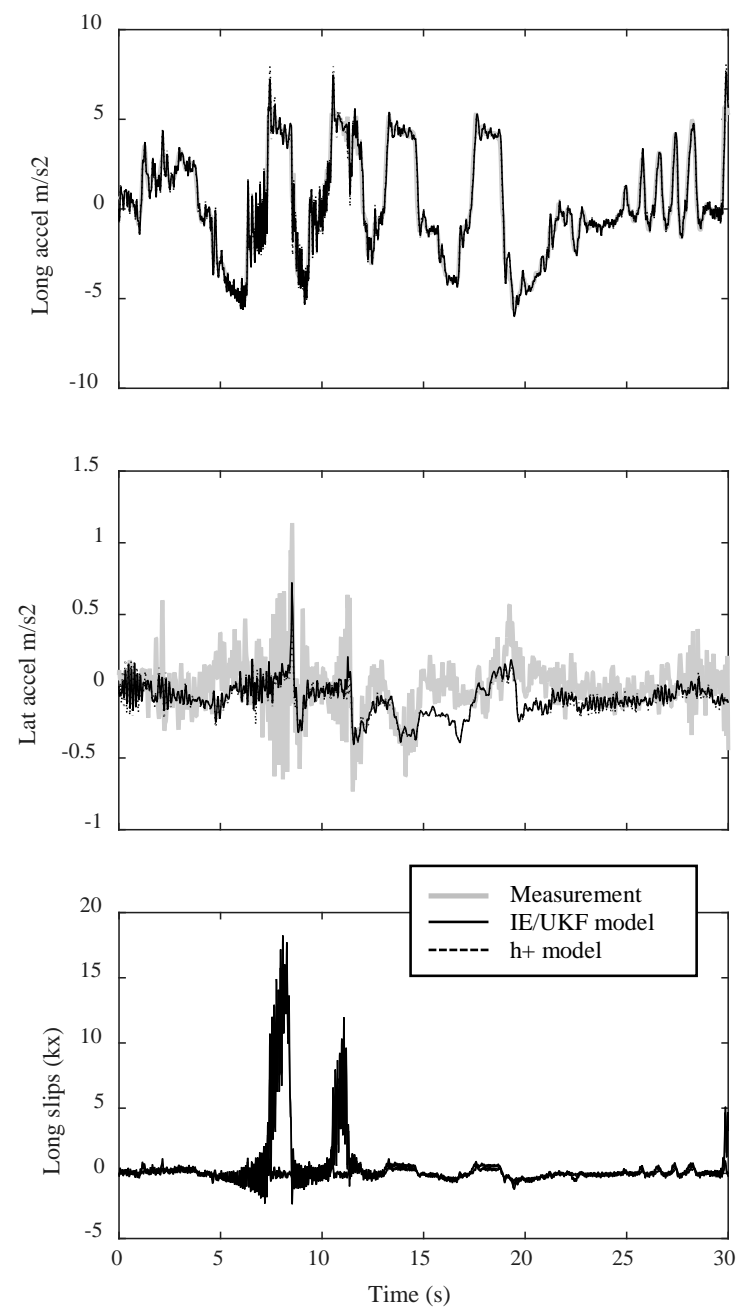

Fig. 5: Model fit to straight-line brake / accel test
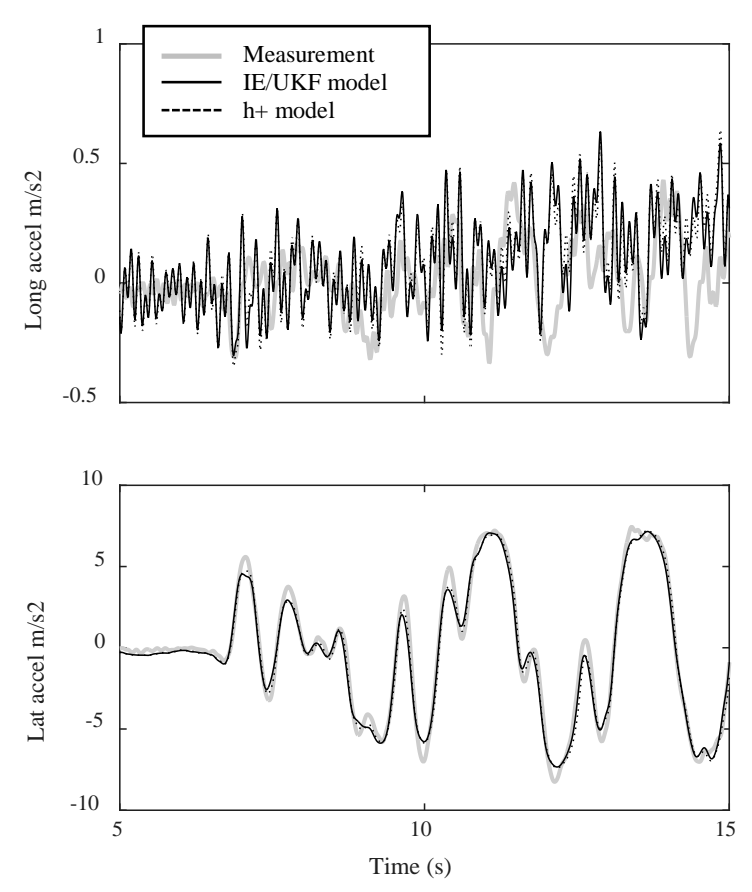

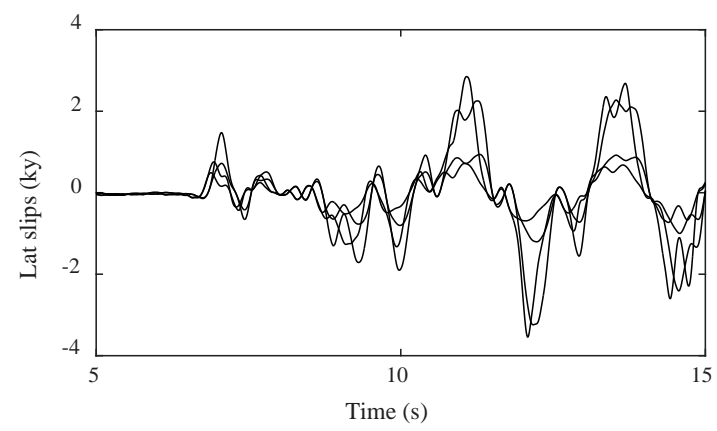

Fig. 6: Model fit to constant speed, random steer test

\section{CONCLUSIONS}

A full set of the independent parameters of a simplified 4-dof nonlinear handling model has been identified, including combined-slip tyre/suspension characteristics identified over their full range up to and beyond saturation. Results show excellent acceleration fits in both single and combined slip validation tests, and the model also performs well on independent output variables.

Unscented and Extended Kalman Filters have been tested, with the UKF proving slightly easier to implement and faster to run, but with both identifying identical models. The choice of which parameters to identify and which to fix can be critical, but the convergence characteristics of the identified parameters make it easy to determine unphysical and/or underdetermined combinations.

\section{REFERENCES}

[1] Wenzel, T.A., et al, "Dual Extended Kalman Filter for Vehicle State and Parameter Estimation”, Vehicle System Dynamics, 2006, Vol. 44, No. 2, pp.153-171.

[2] Best M.C., et al, “An Extended Adaptive Kalman Filter for Real-time State Estimation of Vehicle Handling Dynamics”, Vehicle System Dynamics, 2000, Vol. 34, No. 1, pp. 57-75.

[3] Best, M.C. and Bogdanski, K., "Extending the Kalman Filter for Structured Identification of Linear and Nonlinear Systems”, International Journal of Modelling, Identification and Control. (in press)

[4] Julier, S.J. and Uhlmann, J.K., "A New Extension of the Kalman Filter to Nonlinear Systems", International Society for Optics and Photonics (AeroSense '97), 1997, Vol. 7, No. 28, pp. 182-193.

[5] Wu, Y. et al, "Unscented Kalman Filtering for Additive Noise Case: Augmented vs Nonaugmented”, IEEE Signal Processing Letters, 2005, Vol 12, No. 5, pp. 357-360.

[6] Milliken, D.L. and Milliken, W.F., "Race Car Vehicle Dynamics”, SAE International, 1995

[7] Pacejka, H.B., “Tyre and Vehicle Dynamics”, Butterworth Heinemann, 2002

[8] Best M.C. and Newton A.P., "Vehicle Tyre and Handling Model Identification using an Extended Kalman Filter”, $9^{\text {th }}$ International Symposium on Advanced Vehicle Control (AVEC '08), Taipei, Taiwan, 2008

\section{ACKNOWLEDGMENT}

This work is supported by Jaguar Land Rover and the UK-EPSRC grant EP/K014102/1 as part of the jointly funded Programme for Simulation Innovation (PSi) 